\title{
Cosmological origin of anomalous radio background
}

\author{
James M. Cline* \\ Department of Physics, McGill University, 3600 Rue University, Montréal, Québec, Canada H3A 2T8 \\ Aaron C. Vincent ${ }^{\dagger}$ \\ Instituto de Física Corpuscular, Universitat de València - CSIC, 46071, Valencia, Spain
}

\begin{abstract}
The ARCADE 2 collaboration has reported a significant excess in the isotropic radio background, whose homogeneity cannot be reconciled with clustered sources. This suggests a cosmological origin prior to structure formation. We investigate several potential mechanisms and show that injection of relativistic electrons through late decays of a metastable particle can give rise to the observed excess radio spectrum through synchrotron emission. However, constraints from the cosmic microwave background (CMB) anisotropy, on injection of charged particles and on the primordial magnetic field, present a challenge. The simplest scenario is with a $\gtrsim 9 \mathrm{GeV}$ particle decaying into $e^{+} e^{-}$at a redshift of $z \sim 5$, in a magnetic field of $\sim 5 \mu \mathrm{G}$, which exceeds the CMB $B$-field constraints, unless the field was generated after decoupling. Decays into exotic millicharged particles can alleviate this tension, if they emit synchroton radiation in conjunction with a sufficiently large background magnetic field of a dark $\mathrm{U}(1)^{\prime}$ gauge field.
\end{abstract}

\section{INTRODUCTION}

The ARCADE 2 collaboration has measured the diffuse radio background at several frequencies between 3 and $90 \mathrm{GHz}$, with relatively small errors up to $10 \mathrm{GHz}$ [1]. Their measurements extend earlier ones at lower frequencies $0.01-1 \mathrm{GHz}$ [2]-[5] that are well fit by a power-law dependence of the brightness temperature on frequency,

$$
T \sim T_{R}\left(\nu / \nu_{0}\right)^{\beta}
$$

with $\beta=-2.6 \pm 0.036$. The measured value of $\beta$ is within the ballpark of expectations $(\beta=-2.7)$ for sources in which synchrotron emission dominates (e.g., star-forming galaxies), but the amplitude $T_{R}=1.26 \pm 0.09 \mathrm{~K}$ (with $\nu_{0}=1 \mathrm{GHz}$ ) is $\sim 6$ times too large to be explained by extrapolations of populations of known resolved sources [6]. These conclusions have received further support in ref. [17]. Attempts to identify the excess with previously overlooked standard astrophysical sources have so far not met with success [7]-[9]. There have been several attempts to provide a new origin through the annihilation of dark matter into charged particles, which subsequently undergo synchrotron emission [10]-[14]. It was recently pointed out that there is a serious challenge to such mechanisms: the spatial fluctuations in the observed radio signal are much too small to be consistent with sources that have undergone clustering [15], as would be the case for dark matter annihilating near the present time. ${ }^{1}$ Ref. [15] conservatively uses the linear theory power spectrum for

\footnotetext{
*Electronic address: jcline@physics.mcgill.ca

${ }^{\dagger}$ Electronic address: vincent@ific.uv.es

${ }^{1}$ Ref. [14] studied the possibility of dark matter annihilating into charged particles at earlier times, assuming that ultracompact minihalos and very large magnetic field exist, finding that Compton scattering leads to overproduction of the diffuse x-ray background relative to observed values.
}

its calculations, whereas nonlinear structure formation would be expected to enhance the clustering by a (possibly large) factor that is currently highly uncertain [16]. A possible caveat to the analysis of [15] is its assumption of Gaussian fields in the computation of error bars, whereas the intrinsic fluctuations at the relevant scales are likely dominated by non-linear (hence non-Gaussian) structure. However, the measurements are most likely noise-dominated; thus the statement that clustered dark matter sources are disfavored by the observed smoothness of the signal seems to be robust.

These considerations suggest a cosmological origin of the radio excess, from an epoch prior to the formation of structure and consequent large inhomogeneities in the density of decaying dark matter or of the cosmological magnetic fields. On the other hand, the excess photons should have been produced relatively late, after redshift $z=1100$ when recombination occurred, to avoid thermalizing away such a spectral feature. When one tries to imagine a mechanism for producing excess photons with the right spectrum at this epoch or later, it proves to be highly constrained, due to cosmic microwave background (CMB) constraints $B \lesssim 10^{-9} \mathrm{G}$ on primordial magnetic fields and on injection of ionizing charged particles. The purpose of the present work is to point out some mechanisms that could be promising and the challenges that they must be overcome. We show that a spectrum of excess diffuse radio background consistent with observations can be generated either through synchrotron emission from electrons injected into the plasma at some time after decoupling, depending upon the mass of the decaying particle that produces them. However, CMB constraints on the magnetic field (if it was generated before decoupling) and on the amount of injected energy turn out to be in conflict with this hypothesis.

For example, we find that a metastable particle $\chi$ that decays via $\chi \rightarrow e^{+} e^{-}$can produce the desired radio background through synchrotron emission if $m_{\chi} \gtrsim 700 \mathrm{MeV}$, consistent with the $\mathrm{CMB}$ constraint on ionizing radia- 
tion, but it requires a magnetic field some 100 times greater than the $\mathrm{CMB}$ constraint on primordial $B$ fields, necessitating a late-time magnetogenesis mechanism. A more complicated model may be viable: if $\chi$ decays into millicharged particles that also carry a dark U(1) gauge charge, and if there is a corresponding background of dark photons with a sufficiently large dark magnetic field, the CMB constraint on charged particle injection can be robustly overcome.

Our paper starts with a brief recapitulation of the observed radio spectrum, reviewing in section 2 the data and the possibility that a spectral index -2.5 for the excess temperature provides a consistent description. In section 3 we note that direct production of photons through decays or annihilations does not give this kind of spectrum, nor do processes involving low-energy electrons, motivating the alternative of relativistic charged particle injection. Section 4 discusses the rate of energy loss of charged particles through Compton scattering (CS) and synchrotron emission, and in it we derive an upper bound on the primordial magnetic field around the decoupling epoch, from CMB constraints. This bound implies that CS is by far the dominant means of energy loss. Section 5 computes the spectrum of Comptonscattered photons and consequent bounds from x-ray observations. Section 6 shows that synchrotron emission can produce a radio background with the desired properties, if the initial electron energy is sufficiently large. In sections 5-7 we make a simplifying assumption that the charged particles are injected at specific time (sudden decays). In section 7 we show that this approximation works very well for decays, and we generalize it to the case of annihilations. In section 8 we show that CMB constraints are in conflict with the above mechanisms if the injected particles are electrons, and if the primordial $B$ field was generated before decoupling. In section 9 we suggest a more exotic scenario in which millicharged particles are instead injected, as a second example of how the challenges might be overcome. Conclusions are given in section 10. A possibly novel way of generating primordial magnetic fields (though too weak for our purposes) is elaborated in appendix A. In appendix B we show that brehmsstrahlung cannot yield the observed spectral shape.

\section{THE OBSERVED SPECTRUM}

Taking data from table 4 of ref. [1], which also compiles results from the earlier experiments [2]-[5], we have replotted the antenna temperature ${ }^{2} T_{A}$ data versus frequency and display them in fig. 1. The ARCADE-2 data

\footnotetext{
2 The antenna temperature takes the place of $E /\left(e^{\beta E}-1\right)$ in the familiar formulas for fluxes, intensities, etc., of blackbody radiation. We use units $\hbar=c=k_{B}=1$.
}

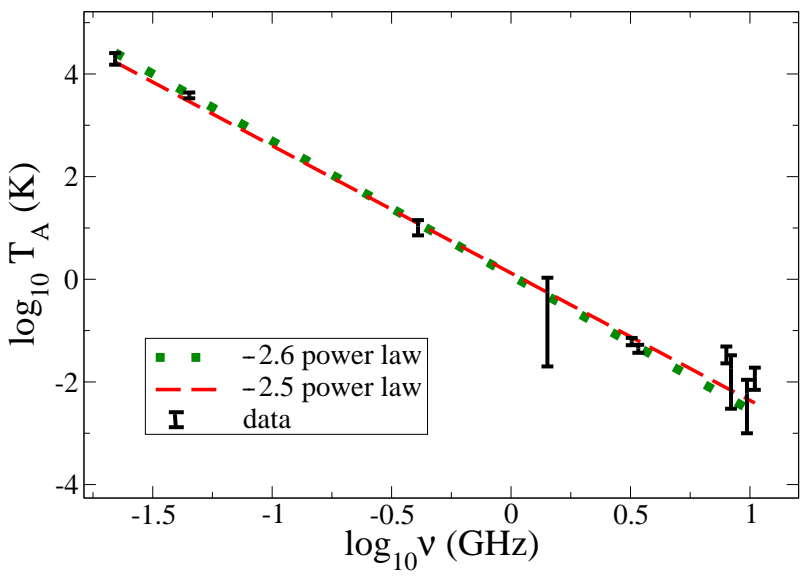

FIG. 1: Excess antenna temperature $T_{A}$ versus frequency as measured by refs. [1]- [5]. Dotted and dashed lines show $\nu^{-2.6}$ and $\nu^{-2.5}$ power laws, respectively.

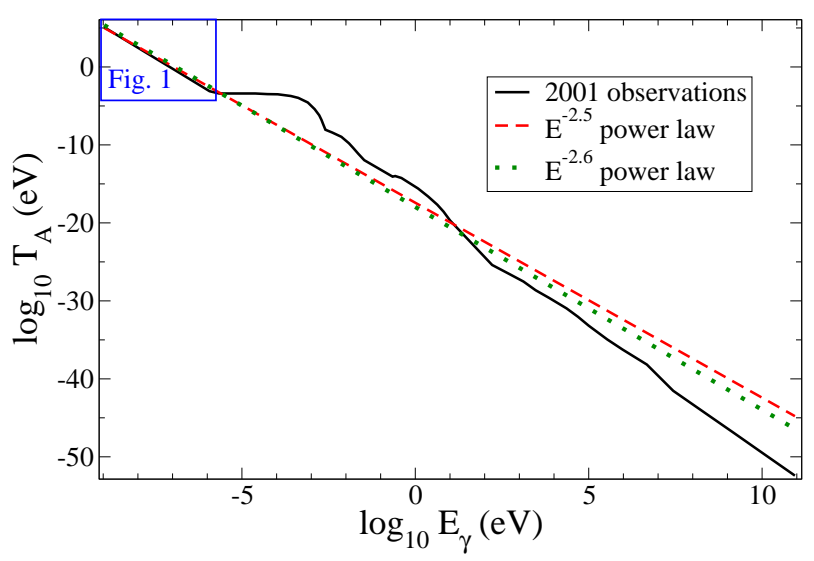

FIG. 2: Temperature of cosmic background radiation to higher frequencies/energies, compared to $E^{-2.6}$ and $E^{-2.5}$ power laws, data taken from fig. 1 of ref. [18]; lines are our fits to the current low-frequency observations. Region of fig. 1 is shown by box in upper left-hand corner.

are the 6 highest frequency points (we do not show those above $10 \mathrm{GHz}$, where the error bars are much larger). Also shown are $\nu^{\beta}$ power law dependences, with the ARCADE- 2 best fit value $\beta=-2.6$, and the theoretical prediction $\beta=2.5$ that we will derive in section 6 . From fig. 1, it is clear that the preference for $\beta=-2.6$ is driven by the measurements near $\nu=45 \mathrm{MHz}$ and 3.3 $\mathrm{GHz}$ that have the smallest errors. This assumes there is no systematic miscalibration between the two different experiments [3] and [1] that determined the respective $T_{A}$ values. Even if not, -2.5 is within $2.8 \sigma$ of ARCADE2 's best-fit value. In the present work, we will consider $\beta=-2.5$ to be adequate for describing the observations. It will be seen that there are more challenging problems for finding a working mechanism than the discrepancy between -2.5 and -2.6 .

To put the radio observations into a broader perspective we have taken the compendium of diffuse radiation 
backgrounds presented in fig. 1 of [18] and translated it into antenna temperature using $T_{A}=4 \pi^{2} \nu^{-2} I_{\nu}$ to indicate how a new source in the radio region (energies less than $\sim 10^{-6} \mathrm{eV}$ ) would compare to higher-energy backgrounds if it continued with the same power law indefinitely. At high energies $E \gtrsim 10 \mathrm{eV}$, the index -2.5 or -2.6 would not be sufficiently steep to remain within observational bounds, but we will see that this need not be a serious limitation. First, the mechanism we propose has a natural cutoff energy above which the spectrum of the new contribution drops sharply, and this can be dialed by adjusting the initial energy of the injected charged particles. Even in the absence of such a cutoff, one must consider whether the universe is transparent to radiation in a given energy range and at a given redshift. The transparency window as a function of $z$ and $E_{\gamma}$ is given in refs. [19, 20]. At low redshifts, this window extends from roughly $1 \mathrm{keV}$ to $10^{5} \mathrm{GeV}$, and it is also open at $E \lesssim 10 \mathrm{eV}$ below the energy of Lyman- $\alpha$ absorption. Thus any x-rays produced in conjunction with the radio excess will be absorbed by the baryonic plasma for energies below $\sim 1 \mathrm{keV}$. At higher redshifts this cutoff increases to $\sim 10 \mathrm{keV}$. Depending upon the details, this effect could weaken the constraining power of experiments like Chandra [21] and XMM-Newton [22] that we will discuss in section 5 .

\section{MOTIVATION FOR RELATIVISTIC CHARGED PARTICLE INJECTION}

One might hope to bypass the complications of synchrotron and Compton emission by a mechanism that directly produces photons after decoupling. An axionlike particle $\chi$ with a very long lifetime and which decays into two photons is the simplest possibility. Although it produces monochromatic photons at any given time, their energies are redshifted and so a continuous spectrum results. Ref. [23] finds, in the case where the decaying particle lifetime $\tau_{\chi}$ is greater than the age of the universe, that the flux of such photons is given by

$\frac{d J}{d E_{\gamma}}=2 \frac{A}{m_{\chi}}\left(1+\kappa\left(\frac{2 E_{\gamma}}{m_{\chi}}\right)^{3}\right)^{-\frac{1}{2}}\left(\frac{2 E_{\gamma}}{m_{\chi}}\right)^{\frac{1}{2}} \Theta\left(1-\frac{2 E_{\gamma}}{m_{\chi}}\right)$

where $A=10^{-7}\left(\mathrm{~cm}^{2} \text { s str }\right)^{-1}\left(10^{17} \mathrm{~s} / \tau_{\chi}\right)\left(10 \mathrm{GeV} / m_{\chi}\right)$ assuming $\chi$ is the dark matter, and $\kappa=\Omega_{\Lambda} / \Omega_{m} \cong 3$. The energy-dependence can be derived from the integral over redshifts $[24]$ up to that at decoupling $\left(z_{\mathrm{dec}}\right)$

$$
\frac{d J}{d E_{\gamma}} \sim \int_{0}^{z_{\mathrm{dec}}} d z \frac{1}{H(z)} \delta\left[\frac{m_{\chi}}{2 E_{\gamma}(1+z)}-1\right]
$$

where the Hubble parameter is proportional to $\sqrt{(1+z)^{3}+\kappa}$ and the delta function yields the spectrum

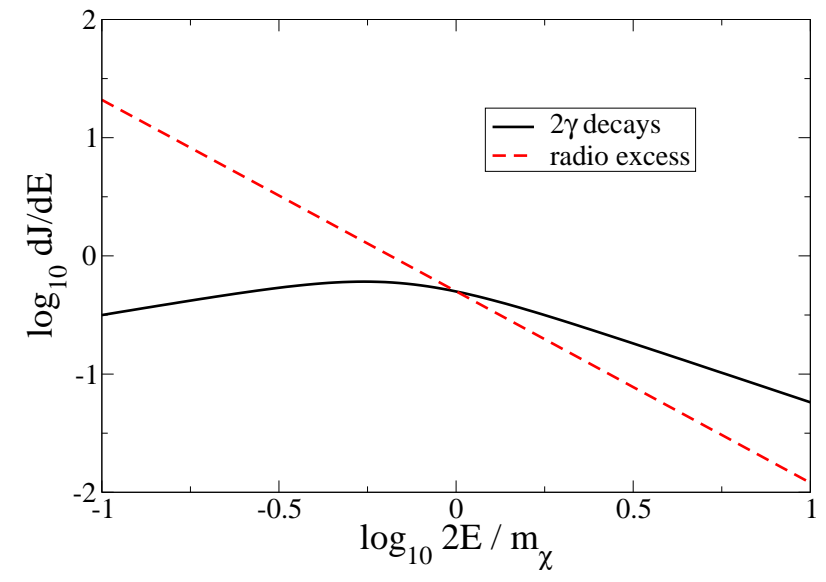

FIG. 3: Comparison of the shape of the observed radio excess flux (in arbitrary units) to that produced by dark matter decays $\chi \rightarrow 2 \gamma$.

of photons from a given decay. ${ }^{3}$

On the other hand, the radio signal of interest has the observed flux

$$
\frac{d J}{d E_{\gamma}}=\frac{E_{\gamma} T\left(E_{\gamma}\right)}{2 \pi^{2}} \sim E_{\gamma}^{-1.6}
$$

where $T\left(E_{\gamma}\right) \cong T_{R} \cdot\left(E_{\gamma} / E_{0}\right)^{-2.6}$ with $E_{0}=4.1 \times 10^{-6} \mathrm{eV}$ (corresponding to $\nu=1 \mathrm{GHz}$ ) and $T_{R}=1.1 \times 10^{-4} \mathrm{eV}$ from (1). The shapes of (2) and (4) are compared in fig. 3 . The slope of the decay spectrum is never as negative as the observed one. We find that the agreement is not improved by considering decays into three photons, approximating the spectral distribution as a box in place of the delta function in (3), nor by decreasing the lifetime such that the $\chi$ 's disappear before the present time (which also changes the shape of the present-day spectrum via an extra factor $e^{-\left(t_{0} / \tau_{\chi}\right)(1+z)^{-3 / 2}}$ in $\left.(3)\right){ }^{4}$ Neither do annihilations $\chi \chi \rightarrow \gamma \gamma$ produce better results, since the input photon spectrum is monochromatic just like for decays. We conclude that particle physics mechanisms for direct production of photons do not work. On the other hand, the radio excess has a spectrum that appears to be consistent with synchrotron emission. Production of charged particles thus seems like a more promising approach.

In order to generate the correct spectrum, we will furthermore require the injected charged particles to be relativistic: $E_{e} \gg m_{e}$. The cyclotron output from nonrelativistic electrons in the cosmic magnetic field will be in the form of peaks around the gyrofrequency. This

\footnotetext{
3 Ref. [24] derives the spectral intensity $I(\lambda)$ rather than the flux, giving an extra factor of $\lambda^{2} / E_{\gamma} \sim(1+z)^{3}$ in the denominator of the integrand.

4 To avoid the problem of large angular fluctuations in the radio excess, we should insist on lifetimes shorter than the time scale for structure formation
} 
is at most $\sim 1 \mathrm{~Hz}$ for values of $B \sim \mathrm{nG}$ allowed by $\mathrm{CMB}$ constraints - far below the $\mathrm{MHz}-\mathrm{GHz}$ region of the ARCADE-2 excess.

Compton scattering with the CMB is a second mechanism which may produce low-energy photons. By computing the lower-limit to the scattered photon energy, we can show that this mechanism is not an efficient means of producing radio photons, if the injected electron is nonrelativistic. A CMB photon with energy $E_{\gamma^{\prime}} \ll m_{e}$ scattering head-on with a low energy electron of velocity $v_{e} \ll 1$ follows the Compton formula, producing a photon of energy $E_{\gamma}$ :

$$
E_{\gamma}=\frac{E_{\gamma^{\prime}}}{1+\sqrt{\frac{1+v_{e}}{1-v_{e}}} \frac{E_{\gamma^{\prime}}}{m_{e}}(1-\cos \theta)},
$$

We are computing an upper limit to energy loss which we therefore maximise by choosing $\cos \theta=0$. This yields:

$$
E_{\gamma}=E_{\gamma^{\prime}}\left(1-\sqrt{\frac{1+v_{e}}{1-v_{e}}} \frac{E_{\gamma^{\prime}}}{m_{e}}+O\left(\left[\frac{E_{\gamma^{\prime}}}{m_{e}}\right]^{2}\right)\right) .
$$

To explain the ARCADE excess, we are seeking a mechanism to produce photons $E_{\gamma} \lesssim 0.01 \bar{E}_{\gamma^{\prime}}$ (the average CMB photon energy). This is clearly not possible in the $v_{e} \ll 1$ regime of $(6)$.

\section{INJECTION OF ELECTRONS AND MAGNETIC FIELD CONSTRAINTS}

We have argued that a more likely mechanism for producing the radio excess would involve injecting charged particles with excess energy into the primordial plasma at some time after recombination but before structure formation, and relying upon the subsequent synchrotron emission. For example, a metastable particle $\chi$ of mass $m_{\chi}>2 m_{e}$ could decay into $e^{+} e^{-}$pairs at the appropriate time. Alternatively a first order phase transition of some scalar field coupled to the Higgs boson could cause a small change in the Higgs vacuum expectation value, leading to a change in the mass of the electron and hence its kinetic energy. However it seems difficult to obtain relativistic electrons as required through the latter mechanism. The decaying $\chi \rightarrow e^{+} e^{-}$scenario on the other hand naturally provides relativistic electrons.

It is enlightening to estimate how much energy is needed to produce the observed excess. Supposing that the spectral anomaly continues up to some maximum energy $E_{\max }$, its energy density is given by

$$
\begin{aligned}
\rho_{r} & =\frac{1}{\pi^{2}} \int_{10^{-2} E_{0}}^{E_{\max }} d E_{\gamma} E_{\gamma}^{2} T\left(E_{\gamma}\right) \\
& \cong 5 \times 10^{-21} \mathrm{eV}^{4}\left(\frac{E_{\max }}{10 E_{0}}\right)^{0.4}
\end{aligned}
$$

(Recall that $E_{0}$ corresponds to the frequency $\nu_{0}=1$ GHz.) As a fraction of the critical density, this is $\Omega_{r}=1.3 \times 10^{-10}\left(E_{\max } / 10 E_{0}\right)^{0.4}$.
Eq. (7) gives a lower bound on the excess kinetic energy in electrons that needs to be injected. But in fact much more is required if we rely upon synchrotron emission, since it is not the most efficient means of dispersing the excess energy in the early universe; Compton scattering on CMB photons $\left(\gamma^{\prime}\right)$ is much more important. The total energy loss rate is due to the sum of the two processes, and their relative importance depends upon the energy density in $\mathrm{CMB}$ photons versus that in magnetic fields:

$$
\frac{d E_{e}}{d t}=\frac{4}{3} \gamma^{2} \sigma_{T}\left(\rho_{\gamma^{\prime}}+\rho_{B}\right)
$$

where $\gamma=E_{e} / m_{e}$ and $\sigma_{T}=(8 \pi / 3) e^{4} / m_{e}^{2}$ is the Thomson cross section. The relative efficiency for producing synchrotron radiation is therefore

$$
\frac{\rho_{B}}{\rho_{\gamma^{\prime}}}=\frac{\frac{1}{2} B^{2}}{\frac{\pi^{2}}{15} T_{\gamma^{\prime}}^{4}} \cong 10^{-7}\left(\frac{B}{10^{-9} \mathrm{G}}\right)^{2}
$$

Here we have alluded to the CMB constraint on the comoving magnetic field $B \lesssim O\left(10^{-9}\right) \mathrm{G}$ [25]-[30]. Taken at face value, a limit of $B<1 \mathrm{nG}$ combined with (7) implies that $10^{7}$ times more energy in Compton photons is produced compared to synchrotron. If we wish to use the latter to produce the radio excess, it would then lead to an extra contribution to $\Omega$ in photons via CS of order $10^{-3}$, which is 25 times greater than that in the CMB.

To understand whether synchrotron emission can be more efficient than indicated by the above discussion requires us to examine the $\mathrm{CMB}$ contraint on $B$ in greater detail. The primordial $B$ field is believed to be stochastic, with a spectrum that in general depends on the length scale. The limit $O\left(10^{-9}\right) \mathrm{G}$ is thus a bound on the average value $B_{\lambda} \equiv\langle B(x) B(y)\rangle^{1 / 2}$ at some comoving scale $\lambda=|x-y|$, which is usually taken to be $1 \mathrm{Mpc}$ for CMB constraints. If the spectrum of $B$ is scale invariant (meaning that $n_{B}=-3$ in $B_{\lambda}^{2} \sim \lambda^{-\left(n_{B}+3\right)}$ ), then there is no possibility that $B_{\lambda}$ could be larger than the nominal bound at some smaller scale. But if it has a blue-tilted spectral index $n_{B}>-3$, then $B_{\lambda}$ could conceivably exceed $O\left(10^{-9}\right) \mathrm{G}$ by some factor at sufficiently small scales (yet large enough to be coherent over the Larmor radius of an electron and thus produce synchrotron radiation).

A limitation is that magnetic fields are damped at small scales by radiative viscosity, and the damping scale is a function of the $B$ field itself. The result of [26] for the damping scale can be expressed as [27]

$$
\frac{\lambda_{D}}{2 \pi} \cong\left(\frac{B_{\lambda}}{170 \times 10^{-9} \mathrm{G}}\right)^{\frac{2}{n+5}}\left(\frac{\lambda}{2 \pi \mathrm{Mpc}}\right)^{\frac{n+3}{n+5}} h^{-\frac{1}{n+5}} \mathrm{Mpc}
$$

(where $n=n_{B}$ ). This applies to vector perturbations, which were shown in ref. [30] to dominate over scalar perturbations. At $B_{1 \mathrm{Mpc}}=10^{-9} \mathrm{G}, \lambda_{D}$ ranges from 0.05 to $0.4 \mathrm{Mpc}$ as $n_{B}$ goes from -3 to 2 (the maximum value usually considered for a causal mechanism of primordial magnetogenesis). For a given $n_{B}$, the maximum field 

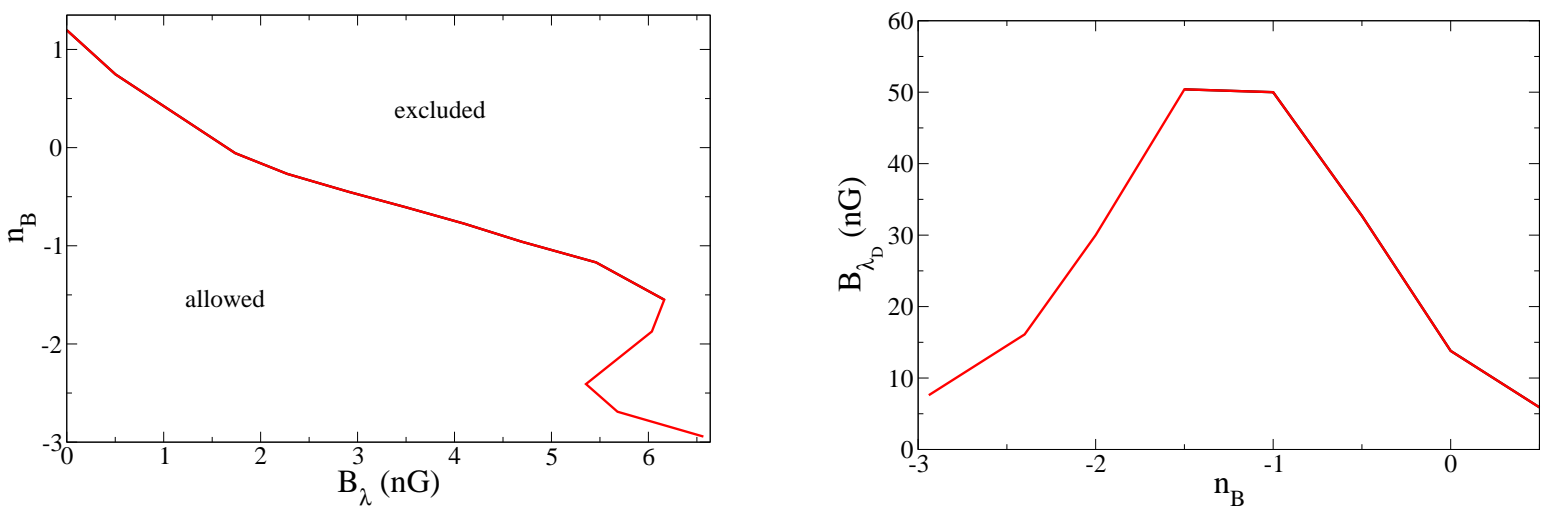

FIG. 4: (a) Left: 95\% C.L. CMB constraints on $n_{B}$ versus $B_{\lambda}$ at $\lambda=1 \mathrm{Mpc}$ from ref. [30]. (b) Right: $B_{\lambda_{D}}$ (i.e., $B_{\lambda}$ at the damping scale (10)) evaluated along the $95 \%$ C.L. contour shown on the left.

value will be attained at the minimum undamped scale, just above $\lambda_{D}$.

CMB constraints on $B_{\lambda}$ and $n_{B}$ are correlated, as shown in ref. [29-31]. We reproduce the $95 \%$ C.L. limit on $n_{B}$ versus $B_{\lambda}$ from ref. [30] in fig. 4(a). The upper limit on $B_{\lambda}$ is strongest for the largest values of $n_{B}$. We have evaluated $B_{\lambda}$ at the damping scale $\lambda_{D}$ for points along this contour to find the maximum allowed $B_{\lambda}$ as a function of $n_{B}$. The result, plotted in fig. 4(b), is that $B_{\lambda_{D}}$ as large as $50 \mathrm{nG}$ is allowed for a spectral index in the range $n_{B}=(-1.5,-1)$, corresponding to damping scales $\lambda_{D}=(0.06-0.1)$ Mpc. Using this field strength in (9), the relative efficiency to produce synchrotron radiation is enhanced by a factor of 2500 , and the energy density produced by associated Compton scattering becomes around 0.01 of that in the $\mathrm{CMB}$, if the excess radio signal is due to synchrotron emission. This relaxation of the nominal bound of $B<$ a few $n G$ is only useful if synchrotron emission occurs close to the decoupling era since thereafter we expect the damping scale to increase again as the free charge density decreases. This caveat will not affect our conclusions in the end since we will have to invoke either late-time magnetogenesis or other exotic particle physics to construct a working scenario.

Stronger limits on $B_{\lambda}$ for scale-noninvariant spectra can be obtained by requiring that the energy density $\rho_{B}$ in $B$ not exceed the critical density at earlier times [32]; the fraction of energy in $\rho_{B}$ increases at earlier times because $\lambda_{D}$ depends upon time (eq. (10) is valid around the epoch of matter-radiation equality). However these limits would be evaded if either the time of magnetogenesis was not too early, or if there is an intrinsic cutoff in the initial spectrum (as one would expect there must be) that falls below the damping scale before $\rho_{B}$ becomes too large. Thus the upper limit $B_{\max } \cong 50 \mathrm{nG}$ is fairly conservative, from the perspective of making the fewest assumptions about the unknown origin of the primordial magnetic field. Of course, if magnetogenesis occurs even later than recombination, we can evade this constraint as well.

A single mechanism that could accomplish both of these at once might interesting for explaining the radio excess. For example, the decay process $\chi \rightarrow e^{+} e^{-}$produces a small electric current at each decay, which gives rise to stochastic $B$ fields. We can estimate their strength as

$$
\langle B(x) B(y)\rangle \sim \frac{4 \pi e^{2} n_{e}}{|x-y|}
$$

for relativistic electrons where $n_{e}$ is the electron density. In appendix A we derive this, and show that it is too small for our purposes. In the following, we will assume that magnetogenesis occurred before decoupling, so the maximum $B$ field was no more than $50 \mathrm{nG}$, although the possibility of relaxing this assumption should be kept in mind if one is looking for loopholes.

\section{COMPTON SCATTERING}

Since we have argued that much more energy will be produced by Compton scattering than by synchrotron emission, it is necessary to compute the spectrum of CS photons resulting from injected electrons. Although this spectrum itself does not have the right properties to explain the excess radio background, it has the potential to make a larger contribution to the diffuse x-ray background than is observed, and thus provides a constraint on mechanisms that rely upon synchrotron emission to account for the radio excess. We derive such a constraint in this section.

The rate of Compton emission of photons of energy $E_{\gamma}$ per scattered electron of initial energy $E_{e} \gg m_{e}$, involves an integral over the energy spectrum $d n / d E_{\gamma^{\prime}}$ of $\mathrm{CMB}$ photons:

$$
\frac{d N_{\gamma}}{d t d E_{\gamma}}=\frac{2 \pi e^{4}}{E_{e}^{2}} \int d E_{\gamma^{\prime}} \frac{1}{E_{\gamma^{\prime}}} \frac{d n}{d E_{\gamma^{\prime}}} f_{C}(q, \Gamma) g_{C}\left(E_{\gamma} / E_{\gamma^{\prime}}\right)
$$

where (following ref. [33])

$$
f_{C}(q, \Gamma)=2 q \ln q+(1+2 q)(1-q)+\frac{(1-q)(\Gamma q)^{2}}{2(1+\Gamma q)}
$$




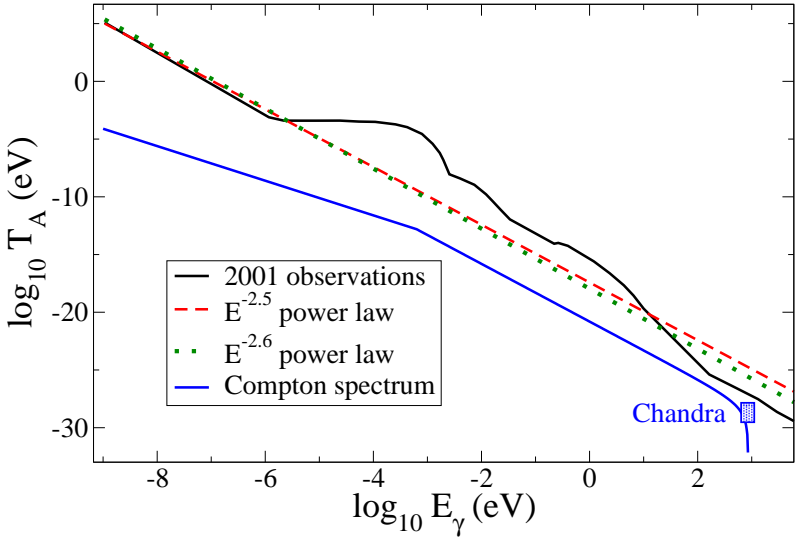

FIG. 5: Solid (blue): the maximum allowed Compton spectrum consistent with Chandra constraint, shown for $m_{\chi}=$ $600 \mathrm{MeV}$. Shaded box shows lowest-energy Chandra observation [21], near edge of transparency window below which $\mathrm{x}$ rays are absorbed by intergalactic medium. Other data taken from fig. 1 of ref. [18]

with $\Gamma=4 E_{\gamma^{\prime}} E_{e} / m_{e}^{2}$ and $q=E_{\gamma} /\left(\Gamma\left(E_{e}-E_{\gamma}\right)\right.$ and

$$
g_{C}\left(E_{\gamma} / E_{\gamma^{\prime}}\right)= \begin{cases}1, & E_{\gamma}>E_{\gamma^{\prime}} \\ E_{\gamma} / E_{\gamma^{\prime}}, & E_{\gamma} \leq E_{\gamma^{\prime}}\end{cases}
$$

The above parametrization is approximate, and can be inferred from fig. 3 of [33]; its main purpose is to show that the spectral index of Compton radiation changes by 1 between the upscattering and downscattering regimes. $f_{C}(q, \Gamma)$, which is meant to describe the regime where $E_{\gamma}>E_{\gamma^{\prime}}$, becomes roughly constant at its maximum value for $E_{\gamma} \leq E_{\gamma^{\prime}}$. This is true provided that the electrons are relativistic. The factor $g_{C}\left(E_{\gamma} / E_{\gamma^{\prime}}\right)$ is designed to give the correct behavior for $E_{\gamma} \leq E_{\gamma^{\prime}}$. We will further approximate (12) by replacing $E_{\gamma^{\prime}}$ with its average value $\bar{E}_{\gamma^{\prime}}=2.7 T_{d}$, and $d n / d E_{\gamma^{\prime}}=n_{\gamma, d} / \bar{E}_{\gamma^{\prime}}$ where $T_{d}$ is the temperature at time when the electron was injected (the decay time $t_{d}$ of $\chi$ in the model where $\chi \rightarrow e^{+} e^{-}$) and $n_{\gamma, d}$ is the density of CMB photons at this temperature. Later on we will relax this "sudden decay approximation" by averaging over $t_{d}$ with the appropriate dependence of $n_{\chi}$ on $t_{d}$. The spectrum of emitted photons at the decay time is then given by

$$
\frac{d n_{\gamma, d}}{d E_{\gamma}} \cong \frac{4 \pi e^{4} n_{\chi, d} n_{\gamma, d}}{\bar{E}_{\gamma^{\prime}}} g_{C}\left(E_{\gamma} / \bar{E}_{\gamma^{\prime}}\right) \int d t E_{e}^{-2} f_{C}(q, \Gamma)
$$

We ignore diffusion since the universe is homogeneous and isotropic during the epoch of interest, and we assume that the electron spectrum remains monoenergetic. The electron energy diminishes very quickly with time; by solving eq. (8) with $\rho_{\gamma^{\prime}} \equiv \rho_{\gamma, d} \gtrsim \rho_{B}$, we find

$$
E_{e}(t)=\frac{E_{d}}{1+C_{e}\left(t-t_{d}\right)}
$$

for $t>t_{d}$, where $E_{d}=m_{\chi} / 2$ is the initial electron energy when it was injected at time $t_{d}$, and

$$
C_{e}=\frac{4}{3} \frac{\sigma_{T} \rho_{\gamma, d} E_{d}}{m_{e}^{2}}
$$

The time scale for energy loss is much smaller than the Hubble time, so we can ignore redshifting of the electron energy. It is convenient to change variable $t \rightarrow \hat{E}_{e}=$ $E_{e} / E_{0}$ in the integral (15), using $d E_{e} / d t=-C_{e} E_{e}^{2} / E_{d}$, where the minimum electron energy is $E_{0} \equiv \frac{1}{2} E_{\gamma}[1+(1+$ $\left.\left.m_{e}^{2} / E_{\gamma} \bar{E}_{\gamma^{\prime}}\right)^{1 / 2}\right]$ from the kinematics of Compton scattering [34]. To evaluate $d n_{\gamma} / d E_{\gamma}$ at the present time, we must correct for redshift by replacing $E_{\gamma} \rightarrow \mathcal{Z}_{d} E_{\gamma}$ and $E_{\gamma^{\prime}} \rightarrow \mathcal{z}_{d} E_{\gamma^{\prime}}$ (where the redshift corresponding to $t_{d}$ is $z_{d} \equiv \mathcal{z}_{d}-1$ ) in $f_{C}$ and in $E_{0}$, and dividing by an overall factor of $\mathcal{Z}_{d}^{2}$ (by dimensionality of $d n_{\gamma} / d E_{\gamma}$ ). Thus

$$
\begin{aligned}
\frac{d n_{\gamma, 0}}{d E_{\gamma}} & =\frac{9 n_{\chi, 0} m_{e}^{4} g_{C}}{8 z_{d}\left(2.7 T_{0}\right)^{2} E_{0}^{3}\left(z_{d}\right)} \int_{1}^{\frac{E_{d}}{E_{0}}} \frac{d \hat{E}_{e}}{\hat{E}_{e}^{4}} f_{C}\left(q\left(z_{d}\right), \Gamma\right) \\
& \cong \frac{9 n_{\chi, 0} m_{e} g_{C}\left(E_{\gamma} / \bar{E}_{\gamma^{\prime}}\right)}{\mathcal{z}_{d}\left(2.7 T_{0}\right)^{1 / 2} E_{\gamma}^{3 / 2}} \times 0.088
\end{aligned}
$$

The approximation 0.088 for the integral holds as long as $E_{d} / E_{0}$ is sufficiently larger than 1 , i.e., for

$$
E_{\gamma} \lesssim 2.6 T_{0}\left(m_{\chi} / m_{e}\right)^{2}
$$

where $T_{0}=2.3 \times 10^{-4} \mathrm{eV}$ is the current CMB temperature. For higher $E_{\gamma}$, the integral drops sharply as shown in fig. 5. To get a contribution at the lowest Chandra energies $E_{\gamma} \cong 0.8 \mathrm{keV}$, for example, would thus require $m_{\chi} \gtrsim 0.6 \mathrm{GeV}$.

At energies below that of the CMB photons, $\bar{E}_{\gamma^{\prime}} / \mathcal{Z}_{d}=$ $2.7 T_{0}$, (18) scales like $E_{\gamma}^{-1 / 2}$ which translates into a subdominant contribution of the form $E_{\gamma}^{-3 / 2}$ to the temperature $T_{A}$ of the excess radio background, where

$$
T_{A}=\frac{\pi^{2}}{E_{\gamma}} \frac{d n_{\gamma}}{d E_{\gamma}}
$$

We can put an upper bound on the initial $\chi$ abundance such that this extra contribution to the radio spectrum does not exceed the observed $E_{\gamma}^{-3 / 2}$ contribution at the highest energies where it is reliably observed. Taking this to be $10 E_{0}=4.1 \times 10^{-5} \mathrm{eV}$, corresponding to frequency $\nu=10 \mathrm{GHz}$, and the corresponding temperature $T_{A} \cong$ $0.0032 \mathrm{~K}=2.8 \times 10^{-7} \mathrm{eV}$ (see fig. 1), we obtain the bound

$$
Y_{\chi}<2 \times 10^{-7}\left(\frac{\mathcal{Z}_{d}}{1100}\right)
$$

on the relative initial abundance of $\chi$ to photons, $Y_{\chi}=$ $n_{\chi} / n_{\gamma}$, before the decays. From (19) one finds that for $m_{\chi}$ greater than a few times $m_{e}$, the Compton contribution to the spectrum extends well above the highest frequency $\sim 90 \mathrm{GHz}$ measured by ARCADE-2 before cutting off. For larger values of $m_{\chi}$ such that the Compton contribution extends into the $\mathrm{x}$-rays, we can derive a much stronger constraint. 
To compare (18) with observations such as those of Chandra [21], we compute

$$
\int I_{\nu} d \nu=\frac{1}{4 \pi} \int_{E_{1}}^{E_{2}} d E_{\gamma} E_{\gamma} \frac{d n_{\gamma, 0}}{d E_{\gamma}} \cong \frac{85 Y_{\chi}\left(\mathcal{z}_{d} / 1100\right)^{-1} \mathrm{erg}}{\mathrm{cm}^{2} \mathrm{~s} \mathrm{deg}^{2}}
$$

in an energy window over which surface brightness has been measured (multiplying by $3 \times 10^{-4}$ to convert from $\operatorname{str}^{-1}$ to $\operatorname{deg}^{-2}$ ). The value $85 Y_{\chi}$ is for the $0.65-1 \mathrm{keV}$ energy interval, in which the measured value is $10^{-12}$ erg $\mathrm{cm}^{-2} \mathrm{~s}^{-1} \mathrm{deg}^{-2}$. We thereby derive a limit on the abundance of decaying $\chi$ particles relative to photons of

$$
Y_{\chi} \lesssim 10^{-14}\left(\frac{\mathcal{Z}_{d}}{1100}\right)
$$

which is much more stringent than (21). However we emphasize that (23) applies only for $m_{\chi} \gtrsim 0.6 \mathrm{GeV}$; otherwise Compton emission in the $E_{\gamma} \sim \mathrm{keV}$ region is kinematically blocked, since then $E_{0}>E_{d}$ and the Compton spectrum cuts off as shown in fig. 5. In this case, a stronger constraint might ostensibly come from lowerenergy x-ray observations, such as from XMM-Newton [22]. But in section 1 we noted that photons of energy lower than $1 \mathrm{keV}$ are effectively attenuated through absorption by hydrogen. Hence the $m_{\chi}<600 \mathrm{MeV}$ restriction is sufficient to generally circumvent the constraints from x-ray observations.

\section{SYNCHROTRON EMISSION}

To derive the spectrum of synchrotron emission from the injected electrons, we follow a similar procedure as for Compton in the previous section. The rate of production of synchrotron photons from a single electron of energy $E_{e}$ is given by [34]

$$
\begin{aligned}
\frac{d N_{\gamma}}{d t d E_{\gamma}} & =\frac{1}{(4 \pi)^{2} \nu} \frac{d E_{\gamma}}{d t d \nu} \\
& =\frac{e^{2} m_{e}^{2}}{\sqrt{3} \pi E_{e}^{2}} F(x)
\end{aligned}
$$

where $x=2 E_{\gamma} m_{e}^{3} /\left(3 e B E_{e}^{2}\right)$ and $F(x)$ is the integral over harmonics of the gyrofrequency of the electron:

$$
\begin{aligned}
F(x) & =\int_{x}^{\infty} K_{3 / 2}(\xi) d \xi \\
& \cong \frac{4 \pi}{3^{\frac{1}{2}} 2^{\frac{1}{3}} \Gamma\left(\frac{1}{3}\right)} x^{-\frac{2}{3}} e^{-x}
\end{aligned}
$$

Although $F(x)$ can be expressed exactly in terms of hypergeometric functions, we find the more tractable approximation in the second line, which is just the known small- $x$ asymptotic value times $e^{-x}$. This is accurate for $x \ll 1$ while overestimating the true value by $\sim 20 \%$ near $x \cong 2.5$ where $F$ is maximized, which is sufficient for our purposes.

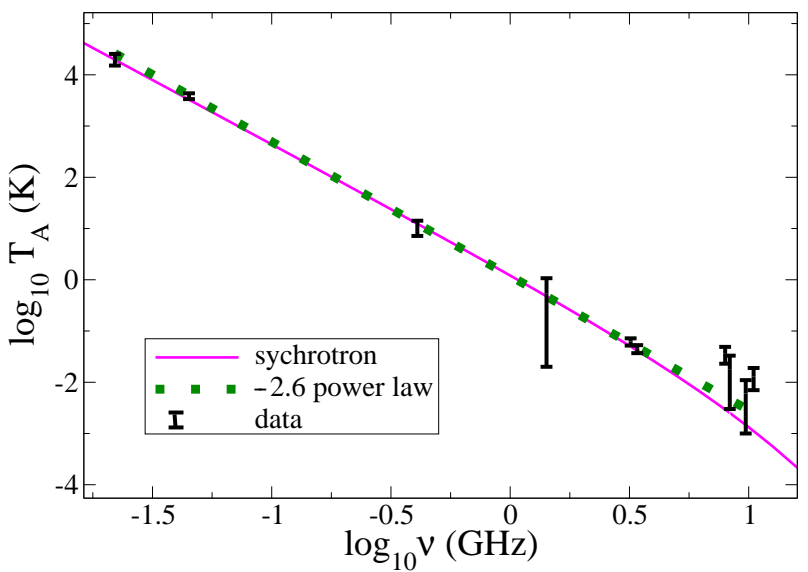

FIG. 6: Solid (magenta): the shape of the predicted contribution to the radio background from synchrotron emission, when the constraint (27) is saturated.

The desired quantity $d n_{e} / d E_{e}$ is obtained by multiplying (24) by the electron density $n_{e}$ and integrating with respect to time. As in the previous section, the relevant time dependence is that of the rapidly decreasing electron energy (16). Again changing variables $t \rightarrow u=x^{2}$ and taking $E_{\gamma} \rightarrow \mathcal{Z}_{d} E_{\gamma}$ (note that also $B \rightarrow \mathcal{z}_{d}^{2} B$ in order to reexpress $B$ at $t_{d}$ in terms of its comoving value), we find

$$
\frac{d n_{\gamma, 0}}{d E_{\gamma}}=\frac{0.048 Y_{\chi} B^{3 / 2} m_{e}^{3 / 2}}{\sqrt{e} \mathcal{Z}_{d}^{3 / 2} T_{0} E_{\gamma}^{3 / 2}} \int_{\sqrt{C_{B}}}^{\sqrt{C_{B}} \frac{E_{d}}{m_{e}}} d u u^{2 / 3} e^{-u^{2}}(26)
$$

where $C_{B}=2 E_{\gamma} m_{e}^{3} /\left(3 e B z_{d} E_{d}^{2}\right)$. We are interested in parameter values such that the upper limit of the integral in (26) is $\gg 1$; in that case the integral can be approximated by $\frac{1}{2}$ the incomplete gamma function $\Gamma\left(\frac{5}{6}, C_{B}\right)$. The latter approaches 1.13 as $C_{B} \rightarrow 0$ but falls exponentially with large $C_{B}$. To insure that $T_{A} \sim E_{\gamma}^{-2.5}$, it is necessary to keep $C_{B} \lesssim 1$ over the relevant range of $E_{\gamma}$. This puts a constraint on the injected energy, here from $m_{\chi}$,

$$
m_{\chi}>6.7 \mathrm{GeV} \times\left(\frac{\mathcal{Z}_{d}}{1100}\right)^{-1 / 2}\left(\frac{B}{50 \mathrm{nG}}\right)^{-1 / 2}
$$

The distortion away from the pure -2.5 power law for the case where $C_{B}=1$, hence (27) is saturated, is shown in fig. 6.

Using $T_{A}=\left(\pi^{2} / E_{\gamma}\right) d n_{\gamma} / d E_{\gamma}$ and normalizing to the measured value at $45 \mathrm{MHz}$, we find that the required relative abundance is

$$
Y_{\chi} \cong 6 \times 10^{-12}\left(\frac{\mathcal{Z}_{d}}{1100}\right)^{3 / 2}\left(\frac{B}{50 \mathrm{nG}}\right)^{-3 / 2}
$$

which is inconsistent with the Chandra constraint (23) unless $B$ exceeds the CMB bound. To make them consistent, we would need $B>0.8 \mu \mathrm{G}\left(z_{d} / 6\right)^{1 / 3}$. Here we have expressed the result with lower redshifts in mind, since this reduces the size of the required field somewhat. This 
assumes that $m_{\chi}>600 \mathrm{MeV}$ so that (23) applies. To evade the x-ray constraint by having $m_{\chi}<600 \mathrm{MeV},(27)$ would require $B$ to be even larger, $B>6.2 \mu \mathrm{G}\left(1100 / \mathcal{Z}_{d}\right)$. However we shall see that $\mathrm{CMB}$ constraints on charged particle injection require yet larger values of $B$ than either of these bounds.

\section{DARK MATTER ANNIHILATIONS AND NONSUDDEN DECAYS}

In the previous sections, for simplicity we considered the case in which electrons are injected suddenly at a specific time $t_{d}$ (the sudden decay approximation). It is straightforward to generalize this to a continuous injection of energy; one replaces $Y_{\chi}$ by

$$
Y_{\chi} \rightarrow \int d t_{d} \frac{d Y_{\chi}}{d t}
$$

(taking into account the time-dependence of $\mathcal{Z}_{d}$ in the expressions for $d n_{\gamma} / d E_{\gamma}$ ) where $d Y_{\chi} / d t$ is the relative abundance per unit time of $\chi$ particles that decay into electrons. The same logic can be applied to annihilations $\chi \chi \rightarrow e^{+} e^{-}$. The expressions for $d Y_{\chi} / d t$ for decays or annihilations are, respectively,

$$
\frac{d Y_{\chi}}{d t}= \begin{cases}Y_{\chi, 0} \tau^{-1} e^{-t / \tau}, & \text { decay } \\ Y_{\chi, 0}^{2} n_{\gamma}\langle\sigma v\rangle, & \text { annihilation }\end{cases}
$$

Let us consider the synchrotron photons coming from annihilation $\chi \chi \rightarrow e^{+} e^{-}$. We can convert the integral over $t_{d}$ into one over $z_{d}$ using $t_{d} \cong t_{0} z_{d}^{-3 / 2}$, which is not accurate at late times when dark energy dominates, but is adequate during matter domination. Carrying out the integral over $z_{d}$, where for a first estimate we approximate $\Gamma\left(5 / 6, C_{B}\right)$ as a step function that goes to zero at $C_{B}=1$, we find that $d n_{\gamma, 0} / d E_{\gamma}$ in (26) is modified by replacing

$$
Y_{\chi} \mathcal{Z}_{d}^{-3 / 2} \rightarrow \frac{3}{2} Y_{\chi, 0}^{2} t_{0} n_{\gamma, 0}\langle\sigma v\rangle \ln \left(\frac{\mathcal{Z}_{\mathrm{dec}}}{\mathcal{Z}_{\mathrm{end}}}\right)
$$

where $\mathcal{Z}_{\text {end }}$ is the value of $\mathcal{Z}_{d}$ such that $C_{B}=1$ and $\mathcal{Z}_{\text {dec }}=1100$. Here $Y_{\chi, 0}$ is the dark matter abundance after it has frozen out, which we can rewrite as $Y_{\chi, 0}=$ $n_{\chi, 0} / n_{\gamma, 0}=2.7 T_{0} \Omega_{\chi} /\left(m_{\chi} \Omega_{\gamma}\right)$, and $\langle\sigma v\rangle$ is the cross section to produce $e^{+} e^{-}$. It is therefore no more than $\left(\Omega_{D M} / \Omega_{\chi}\right)\langle\sigma v\rangle_{0}$, where $\langle\sigma v\rangle_{0}=3 \times 10^{-26} \mathrm{~cm}^{3} / \mathrm{s}$ is the standard thermal cross section for obtaining the observed $\Omega_{D M}=0.22 .{ }^{5}$ Here we only assume that $\Omega_{\chi}<0.22$ since $\chi$ need not be the only species of dark matter. Now

\footnotetext{
${ }^{5}$ If there is some other annihilation channel determining the $\chi$ relic density, then $\langle\sigma v\rangle$ for $\chi \rightarrow e^{+} e^{-}$can be smaller than this bound.
}

equating the modified (26) to the observed radio spectrum, we find the necessary condition (but not sufficient, as will become immediately apparent)

$$
m_{\chi}=0.6 \mathrm{MeV} \times \sqrt{r}\left(\frac{\Omega_{\chi}}{\Omega_{D M}}\right)^{1 / 2}\left(\frac{B}{50 \mathrm{nG}}\right)^{3 / 4}
$$

where we define $r$ to be the ratio of $\langle\sigma v\rangle$ for $\chi \chi \rightarrow e^{+} e^{-}$ to the total annihilation cross section. For simplicity we assume that any additional annihilation channels do not result in charged particles, since otherwise these will quickly decay into electrons and behave similarly to the electrons produced as primary annihilation products. The requirement (32) insures that the spectrum matches the observed one at arbitrarily low frequencies, but it does not take into account the need for the highfrequency cutoff to extend to the observed $\mathrm{GHz}$ region. This requires $\ln \left(\mathcal{Z}_{\text {dec }} / \mathcal{Z}_{\text {end }}\right) \sim 1$, which is only consistent if $\mathcal{Z}_{\text {end }} \lesssim \mathcal{Z}_{\text {dec }}$, which puts a second constraint on the mass (see (27)),

$$
m_{\chi}>6.7 \mathrm{GeV} \times\left(\frac{B}{50 \mathrm{nG}}\right)^{-1 / 2}
$$

These constraints begin to overlap (leaving no allowed parameter space) if the field is too small, leading to the lower bound

$$
B>90 \mu \mathrm{G} \times\left(\frac{\Omega_{D M}}{r \Omega_{\chi}}\right)^{2 / 5}
$$

In the case where this bound is saturated, the mass is $m_{\chi}=160\left(r \Omega_{\chi} / \Omega_{D M}\right)^{1 / 5} \mathrm{MeV}$. We will see that this is strongly ruled out by CMB constraints on charged particle injection. Thus dark matter annihilations in the early universe cannot explain the radio excess even with late-time magnetogenesis.

For decays, we can carry out the time-averaging to find the more accurate dependence of the normalization of the spectra upon $\mathcal{Z}_{d}$. This results in a correction factor of order unity:

$$
\frac{1}{\mathcal{Z}_{d}^{p}} \rightarrow \frac{1}{\mathcal{Z}_{d}^{p}} \int_{t_{\mathrm{dec}} / \tau}^{t_{0} / \tau} d x x^{2 p / 3} e^{-x}
$$

where $t_{\mathrm{dec}}=3.8 \times 10^{5} \mathrm{y}$ is the age of the universe at decoupling, and $t_{0}=13.8 \mathrm{Gyr}$ is the present age. For $t_{\text {dec }} \ll \tau \ll t_{0}$, the correction factor is approximately $\Gamma(1+2 p / 3)=0.9,1$ for $p=1,3 / 2$, corresponding to the cases of Compton and synchrotron emission, respectively. Thus the sudden approximation works quite well for the case of decays when $\tau$ is in the range of interest.

\section{CMB CONSTRAINTS ON CHARGED PARTICLE INJECTION}

There are stringent bounds on the injection of ionizing energy after decoupling due to its distortion of the CMB 
anisotropies, either through particle decays [19, 35] or annihilations $[20,36]$. These depend mainly upon the total amount of kinetic energy of charged particles that is injected, and so are closely correlated with the requirements for producing the observed radio background. We first consider the case of decaying particles. Ref. [35] obtains

$$
\frac{Y_{\chi}}{\tau} \lesssim 2 \times 10^{-25} \mathrm{~s}^{-1} Y_{D M}
$$

for $\tau>10^{17} \mathrm{~s}$, and a somewhat less stringent limit at shorter lifetimes; however the above approximation will be adequate for our purposes since the tension of this constraint with what is needed for the radio excess is minimized at the higher values of $\tau$, despite the modest weakening of the constraint at low $\tau$. Here $Y_{D M}=\left(\Omega_{D M} / \Omega_{\gamma^{\prime}}\right)\left(\bar{E}_{\gamma^{\prime}} / m_{\chi}\right)$ is the standard abundance that $\chi$ would have if it was a stable particle constituting the full WMAP DM abundance. Substituting $\mathcal{Z}_{d}$ for $\tau$ we then find

$$
Y_{\chi}<2 \times 10^{-17}\left(\frac{6}{\mathcal{Z}_{d}}\right)^{3 / 2}\left(\frac{1 \mathrm{GeV}}{m_{\chi}}\right)
$$

We have taken $\mathcal{Z}_{d}=6$ to be the latest decay epoch consistent with generating the signal before significant clustering of the DM would occur and induce unwanted fluctuations. If we take the synchrotron mass bound (27) to be saturated, then (37) becomes $Y_{\chi}<$ $10^{-18}\left(6 / \mathcal{Z}_{d}\right)^{1 / 2}(B / 1 \mu \mathrm{G})^{1 / 2}$. Comparing this to the required value of $Y_{\chi}(28)$, we get the lower bound on $B$

$$
B>5 \mu \mathrm{G} \times\left(\frac{\mathcal{Z}_{d}}{6}\right)^{5 / 4}
$$

For this value of $B$, the minimum $\chi$ mass from (27) is $m_{\chi}=9 \times\left(z_{d} / 6\right)^{-9 / 8} \mathrm{GeV}$, and the abundance is $Y_{\chi}=2 \times 10^{-18}\left(z_{d} / 6\right)^{-3 / 8}$. This is compatible with the x-ray constraint (23), which can be written as $Y_{\chi}<$ $5 \times 10^{-17}\left(z_{d} / 6\right)$. A summary of the bounds on electron injection from $\chi$ decay is presented in Figure 7 .

For $\chi \chi \rightarrow e^{+} e^{-}$annihilations, we adapt the result of ref. [20] to obtain

$$
\langle\sigma v\rangle \equiv r\langle\sigma v\rangle_{\text {tot }} \lesssim f^{-1}\langle\sigma v\rangle_{0}\left(\frac{m_{\chi}}{10 \mathrm{GeV}}\right)\left(\frac{\Omega_{D M}}{\Omega_{\chi}}\right)^{2}
$$

where $f$ is an efficiency factor that is close to 1 for annihilation into electrons, and $r$ was defined below eq. (32). Since the rate of injection of charged particles depends upon $n_{\chi}^{2}$, we have appended the factor $\left(\Omega_{D M} / \Omega_{\chi}\right)^{2}$ for the case $\Omega_{\chi}<\Omega_{D M}$ to account for the weakening of the bound derived by [20], where it was assumed that $\Omega_{\chi}=$ $\Omega_{D M}$. Consider the case where the $B$ field is just barely large enough to give the radio excess, so that (34) is saturated. Then using the fact that $\langle\sigma v\rangle_{\text {tot }} /\langle\sigma v\rangle_{0}=\Omega_{D M} / \Omega_{\chi}$ and the value of $m_{\chi}$ determined below eq. (34), we find that the CMB constraint can be written as

$$
\left(\frac{r \Omega_{\chi}}{\Omega_{D M}}\right)<6 \times 10^{-3}
$$

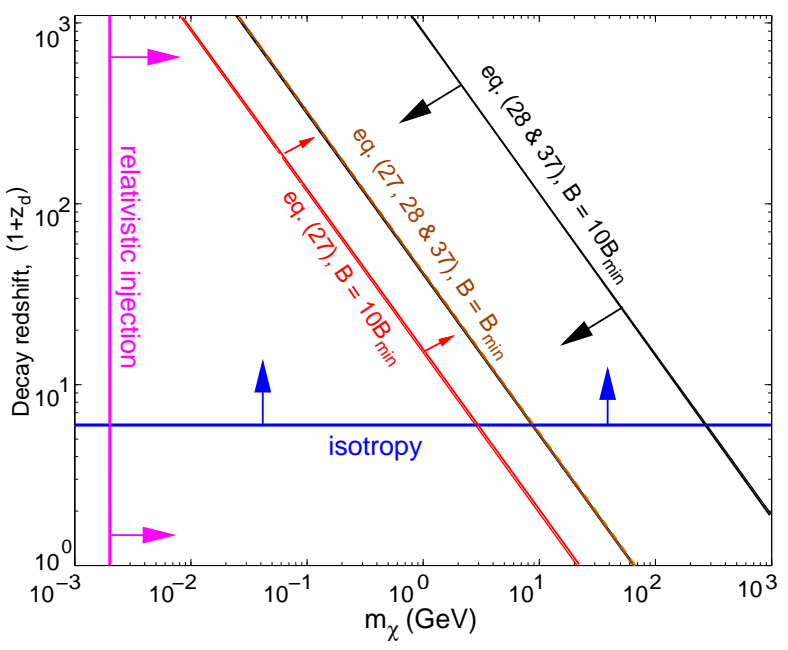

FIG. 7: Summary of constraints on models with electron injection at redshift $z_{d}$ from $\chi \rightarrow e^{+} e^{-}$decays, as a function of $m_{\chi}$. Allowed regions are in directions of arrows. "Isotropy" (blue) denotes the limit $z_{d}>5$ due to the isotropy of the signal. "Relativistic injection" (magenta) denotes requirement limit $m_{\chi} \gtrsim 2 \mathrm{MeV}$ in order to obtain relativistic electrons. The "eq. (27, 28 \& 37)" line (brown) is the coincidence of upper and lower limits on $m_{\chi}$ in the case of minimum $B$ field given by eq. (38). If $B$ is allowed to be 10 times larger than this minimum value, the allowed region is bounded by the "eq. (27)" (red) and "eq. (28 \& 37)" (black) curves, coming from the shape of the synchrotron spectrum and the CMB limit on charged particle injection, respectively.

which with eq. (34) then implies $B>3 \mathrm{mG}$ (milligauss) in order for annihilations to give the radio excess. This is such a strong field that it would overclose the universe.

\section{MILLICHARGED MODEL}

If we insist that $B$ was generated prior to decoupling and thus must obey the CMB constraints, additional new physics is required. In this section we present a somewhat more complicated model, in which the injected particles, instead of being electrons, are exotic particles that we denote by $\tilde{e}$, carrying a fractional electric charge $\epsilon e$. In adddition, we assume that $\tilde{e}$ has a larger charge $g$ under a hidden, unbroken $U(1)^{\prime}$ gauge group that comes with its own "dark photons," (denoted by $\tilde{\gamma}$ ) having a cosmological background analogous to the CMB. This scenario has been considered previously in the millicharged atomic dark matter model of ref. [37, 38].

It is straightforward to generalize the Compton result (18) to the present model. For the rate of production of CS photons, the combination $e^{4} n_{\gamma} \bar{E}_{\gamma^{\prime}}^{1 / 2}$ coming from $e^{4} n_{\gamma} /\left(\bar{E}_{\gamma^{\prime}} E_{0}^{3}\right)$ is replaced by

$$
A \equiv g^{2}(\epsilon e)^{2} n_{\tilde{\gamma}} \bar{E}_{\tilde{\gamma}}^{1 / 2}+(\epsilon e)^{4} n_{\gamma} \bar{E}_{\gamma^{\prime}}^{1 / 2}
$$


On the other hand in the energy loss rate that eventually appears in the denominator, the combination $e^{4} \rho_{\gamma}$ is replaced by

$$
A^{\prime}=g^{4} \rho_{\tilde{\gamma}}+g^{2}(\epsilon e)^{2}\left(\rho_{\tilde{\gamma}}+\rho_{\gamma}\right)+(\epsilon e)^{4} \rho_{\gamma}
$$

Thus the CS spectrum will be proportional to $A / A^{\prime}$. In addition the electron mass is replaced by $m_{\tilde{e}}$. Assuming that the first term in each of (41) and (42) dominates, we find that the x-ray constraint (23) generalizes to

$$
Y_{\chi}<5 \times 10^{-17}\left(\frac{\mathcal{Z}_{d}}{6}\right)\left(\frac{\tilde{T}_{0}}{T_{0}}\right)^{1 / 2}\left(\frac{g}{\epsilon e}\right)^{2}\left(\frac{m_{e}}{m_{\tilde{e}}}\right)
$$

In contrast, the CMB constraint depends mainly upon the fraction of ionizing electromagnetic energy per decay. In the present model, this fraction is given by $\left(A^{\prime}-g^{4} \rho_{\tilde{\gamma}}\right) / A^{\prime}$. The bound (37) is accordingly weakened to become

$$
Y_{\chi}<2 \times 10^{-17}\left(\frac{6}{\mathcal{Z}_{d}}\right)^{3 / 2}\left(\frac{1 \mathrm{GeV}}{m_{\chi}}\right)\left(\frac{g}{\epsilon e}\right)^{2}\left(\frac{\tilde{T}_{0}^{4}}{T_{0}^{4}}\right)
$$

where we have assumed that the leading terms in numerator and denominator of the reduction factor are dominant, and also that $\tilde{T}_{0}^{4} \ll T_{0}^{4}$. These two assumptions would be consistent if for example $\tilde{T}_{0}^{4} \sim 0.1 T_{0}^{4}$, the maximum allowed by constraints on the Hubble rate during big bang nucleosynthesis. We need to choose the more restrictive of the two bounds (43) and (44) in what follows.

The millicharged model can also admit a primordial dark magnetic field $\tilde{B}$ whose magnitude is less constrained than that of the visible $B$ field, allowing for the possibility of early $\tilde{B}$ magnetogenesis that is unconstrained by the CMB. The modified synchrotron spectrum can be deduced from the derivation of section 6 by making the following changes. (1) $e \rightarrow \epsilon e$ for the rate of emission in (24); (2) $e B \rightarrow g \tilde{B}$ in the definition of $x$, since the cyclotron frequency is now determined by this combination; (3) $e \rightarrow g$ in the Thomson cross section and $\rho_{\gamma} \rightarrow \rho_{\tilde{\gamma}}$ coming from the energy loss rate, which is dominated by $\tilde{\gamma}$ Compton emission; (4) obviously $m_{e} \rightarrow m_{\tilde{e}}$ everywhere as well. In this way, we find that the required abundance (28) generalizes to

$$
\begin{aligned}
Y_{\chi, \text { sync }}= & 2.7 \times 10^{-17} \times \\
& \epsilon^{-2}\left(\frac{\tilde{T}_{0}}{T_{0}}\right)^{4}\left(\frac{\mathcal{Z}_{d}}{6}\right)^{\frac{3}{2}}\left(\frac{\tilde{B}}{1 \mu \mathrm{G}}\right)^{-\frac{3}{2}}\left(\frac{m_{e}}{m_{\tilde{e}}}\right)^{\frac{3}{2}}\left(\frac{g}{e}\right)^{\frac{5}{2}}
\end{aligned}
$$

which is valid as long as $g^{4} \tilde{T}_{0}^{4} \gtrsim(g \epsilon e)^{2} T_{0}^{4}$. Similarly, the generalization of the mass constraint (27) is

$$
m_{\chi} \gtrsim 20 \mathrm{GeV}\left(\frac{\mathcal{Z}_{d}}{6}\right)^{-\frac{1}{2}}\left(\frac{\tilde{B}}{1 \mu \mathrm{G}}\right)^{-\frac{1}{2}}\left(\frac{e}{g}\right)^{\frac{1}{2}}\left(\frac{m_{\tilde{e}}}{m_{e}}\right)^{\frac{3}{2}}
$$

Proceeding as in section 8, by inserting (46) into (44) and comparing to (45), we find that all the dependence upon new parameters cancels out and yields (38) again, but now as a constraint on $\tilde{B}$ instead of $B$. The advantage is that there are relatively few direct constraints on the dark $\tilde{B}$ field, and it could therefore have been generated prior to decoupling. For example taking $\mathcal{Z}_{d}=6$, $m_{\chi}=5 \times\left(m_{\tilde{e}} / m_{e}\right)^{3 / 2} \mathrm{GeV}, \tilde{B}=5 \mu \mathrm{G}, \epsilon=10^{-3}, g=$ $\sqrt{10} e$, we find from $(45)$ that $Y_{\chi}=4 \times 10^{-12}\left(m_{e} / m_{\tilde{e}}\right)^{3 / 2}$, which marginally satisfies the CMB constraint (44) for any value of $m_{\tilde{e}}$. We can then take $m_{\tilde{e}}>100 \mathrm{MeV}$ (hence $m_{\chi}>14 \mathrm{TeV}$ ) and be consistent with constraints on millicharged particles [39-41]. The x-ray constraint (43) is weaker than (44) and provides no additional information. For such values of $Y_{\chi}$, the mass density of $\chi$ is sufficiently small before it decays, $\Omega_{\chi} \sim 0.04 \Omega_{b}$, that there is no danger of it significantly affecting the expansion history of the universe.

\section{CONCLUSIONS}

The diffuse excess radio background observed by the ARCADE-2 collaboration has a spectrum that appears to be consistent with observations of four other groups. This adds to the plausibility of it being a real anomaly, and the observation of ref. [15] concerning its homogeneity seems to constitutes a strong motivation for its origin to be cosmological in nature, in the absence of ubiquitous astrophysical sources of size $>1 \mathrm{Mpc}$.

Using approximate analytic methods, ${ }^{6}$ we have shown that injection of relativistic electrons after decoupling, through late decays of a metastable particle $\chi$, can give rise to a radio excess with the correct power law falloff through synchrotron emission. However we find that injecting enough charge to get the right normalization of the excess background should also have distorted the CMB fluctuations relative to their observed properties, due to the deposition of electromagnetic energy into the intergalactic medium. This contradiction can be circumvented if the primordial $B$ field is larger than expected on the basis of other CMB constraints, $B>5 \mu \mathrm{G}$. The size of the required field is smallest when the decays are as late as possible (but still before the onset of structure formation, to avoid large fluctuations in the radio background). Such large fields would have to be generated by some unknown cosmological mechanism after decoupling in order to avoid the CMB $B$-field constraint, $B \lesssim$ several $\mathrm{nG}$.

Based on our results, the simplest and most conservative cosmological scenario for producing the excess radio background is through the synchroton radiation emitted

\footnotetext{
${ }^{6}$ notably the approximation of (25) and extending the upper limit of integration in (26). We expect this to introduce errors of order $20 \%$ in the normalization of the spectrum, and $\mathrm{O}(1)$ errors in the details of the spectrum in the high-frequency region where it is rapidly falling away from the -2.5 power law behavior.
} 
by electrons from decays $\chi \rightarrow e^{+} e^{-}$at redshift $z=5$, in a magnetic field of strength $B \sim 5 \mu \mathrm{G}$, with $m_{\chi} \gtrsim 9 \mathrm{GeV}$ and an initial abundance $Y_{\chi} \sim 10^{-18}$ relative to photons. Earlier decay epochs would require larger $B$ fields, given by (38). This requires magnetogenesis at low redshifts $z<1100$ to circumvent CMB constraints on the primordial magnetic field. We find that the annihilation scenario, $\chi \chi \rightarrow e^{+} e^{-}$, is too inefficient and would require unacceptably large $B$ fields to produce the observed radio excess.

One might wonder whether decays into other known charged particles than the electron could give more promising results, since the CMB bound is weakened in that case. The weakening comes about because some of the initial energy eventually goes into neutrinos, which do not ionize the intergalactic medium. We do not expect any improvement in this way however, since the same loss of efficiency in the production of radio and other electromagnetic backgrounds should occur, and the amount of radiation produced by the unstable charged particles before they turn into electrons must be very small due to their short lifetimes, compared to the time scale for energy loss through CS.

To demonstrate an alternative example that does not require late-time magnetogenesis, we presented a model of small fractionally charged $\left(\sim 10^{-3} e\right)$ decay products with mass $\gtrsim 100 \mathrm{MeV}$, produced from the decays of heavy $\gtrsim 14 \mathrm{TeV}$ parent particles. If the fractionally charged particles undergo cyclotron motion due to their interaction with a dark background magnetic field $\tilde{B} \gtrsim 5 \mu \mathrm{G}$, the resulting synchrotron emission (in normal photons) can explain the radio anomaly without conflicting with CMB observations. The fractionally charged particles are stable relics, but with an abundance less than $10^{-5}$ that of baryons, making them innocuous at present times. This example might be regarded as proof that it is possible to construct a viable, albeit exotic model; we hope it will motivate others to search for alternative possibilities. ${ }^{7}$

Acknowledgments. We are grateful to F. Tavecchio for pointing out an important error in the first version of this manuscript. We thank M. Cirelli, R. Durrer, F. Finelli, M. Giovannini, G. Holder, Z. Liu, T. Kahniashvili, M. Kamionkowski, G. Moore, V. Poitras, P. Scott and T. Slatyer for helpful dicussions or correspondence. Our work was supported by the Natural Sciences and Engineering Research Council (NSERC, Canada). AV is supported by European contract PITN-GA-2011-289442INVISIBLES and Fonds de recherche du Québec - Nature et technologies (FQRNT).

\footnotetext{
7 Recently ref. [42] appeared, which also proposes a cosmological dark matter mechanism.
}

Appendix A: Stochastic $B$ field from $\chi \rightarrow e^{+} e^{-}$decay

In this appendix we estimate the stochastic magnetic field spectrum that is generated from the decays of a heavy particle $\chi$ into $e^{+} e^{-}$. Consider a decay at time $t=$ 0 and position $\vec{r}=0$, producing an electron of velocity $\vec{v}$. The vector potential due to the $e^{+} e^{-}$pair is

$$
\vec{A}=e \vec{v}\left(|\vec{r}-\vec{v} t|^{-1}-|\vec{r}+\vec{v} t|^{-1}\right)
$$

We can compute the desired correlation function $\left\langle B_{i}(x) B_{j}(y)\right\rangle$ from $\left\langle A_{i}(\vec{x}) A_{j}(\vec{y})\right\rangle$ by taking the appropriate curls. The correlators can be found by averaging over a random ensemble of vector potentials due to currents emanating from arbitrary points in arbitrary directions (but for simplicity we take them all to arise at the same time). We take the contributions from different currents to be uncorrelated so that only diagonal terms in the product of the two $A$ 's contribute. The correlator thus becomes a sum over all particles, averaging over the position of the decaying parent and the direction of the products. Let $N$ be the total number of decaying particles in a volume $V$, and define $\vec{x}_{ \pm}=\vec{x}-\left(\vec{x}_{0} \pm \vec{v} t\right)$ where $x_{0}$ is the initial position of a fiducial pair; similarly for $\vec{y}_{ \pm}$. Then

$$
\begin{aligned}
\left\langle A_{i}(\vec{x}) A_{j}(\vec{y})\right\rangle=e^{2} N\left\langle v_{i} v_{j}\right. & \left(\left|\vec{x}_{+}\right|^{-1}-\left|\vec{x}_{-}\right|^{-1}\right) \\
& \left.\left.\times\left(\left|\vec{y}_{+}\right|^{-1}-\left|\vec{y}_{-}\right|^{-1}\right)\right)\right\rangle
\end{aligned}
$$

The averaging means

$$
\langle X\rangle=\frac{1}{4 \pi V} \int d^{3} x_{0} \int d \Omega_{v} X
$$

The integral over $x_{0}$ can be done by expressing

$$
\left|\vec{x}_{ \pm}\right|^{-1}=\frac{1}{\sqrt{\pi}} \int_{0}^{\infty} \frac{d s_{ \pm}}{\sqrt{s_{ \pm}}} e^{-s_{ \pm}\left|\vec{x}_{ \pm}\right|^{2}}
$$

The integrals over $s_{ \pm}$can also be done exactly, leading to

$$
\left\langle A_{i}(\vec{x}) A_{j}(\vec{y})\right\rangle=e^{2} n_{e} \int d \Omega_{v} v_{i} v_{j}\left(|\vec{x}-\vec{y}|-\left|\vec{x}_{+}-\vec{y}_{-}\right|\right)
$$

where $n_{e}$ is the electron density. The remaining integral over angles can also be done exactly, but at large times the leading contribution to the gradients will be from the first term, which is given by

$$
\left\langle A_{i}(\vec{x}) A_{j}(\vec{y})\right\rangle \cong 4 \pi e^{2} v^{2} n_{e} \delta_{i j}|\vec{x}-\vec{y}|
$$

The $B$ field correlator thus goes like $1 /|\vec{x}-\vec{y}|$, corresponding to a spectral index of $n_{B}=-2$ in the notation of section 4 .

The smallest scale that can be relevant for synchrotron emission is the Larmor radius $r=m_{e} c / e B$. Eliminating $r$ from this equation and (11) at $r=|x-y|$, we find the field strength $B=4 \pi e^{3} n_{e} / m_{e}$ (evaluated at the decay 
redshift $z_{d}<1100$; this value of $B$ must be divided by $z_{d}^{2}$ to convert it to the comoving value for comparison with the CMB constraint). Even if the electrons are injected with unit abundance relative to photons, this is orders of magnitude smaller than the CMB limit and thus irrelevant.

\section{Appendix B: Brehmsstrahlung}

The final mechanism for photon production from charged particles is by Bremsstrahlung with the ambient neutral (before reionization) or charged (after) hydrogen atoms. We obtain the photon spectrum in the same way as before, by assuming a sudden decay, and replacing the integral over time with an integral over the electron's energy, which is lost due to Compton scattering. The bremsstrahlung spectrum of a relativistic electron colliding with a hydrogen atom is [34]:

$$
\begin{aligned}
\frac{\mathrm{d} N_{\gamma}}{\mathrm{d} E_{\gamma} d t} & =\frac{\alpha r_{0}^{2}}{E_{\gamma} E_{e}^{2}}\left[\left(E_{e}^{2}+\left(E_{e}-E_{\gamma}\right)^{2}\right) \phi_{1}\right. \\
& \left.-\frac{2}{3} E_{e}\left(E_{e}-E_{\gamma}\right) \phi_{2}\right]
\end{aligned}
$$

where $r_{0}$ is the classical electron radius and $E_{e}-E_{\gamma}$ is approximately the electron energy after scattering. The functions $\phi_{i}$ parametrize the atomic shielding. When $E_{\gamma} \ll E_{e}$, there are two possibilities: 1) scattering off unshielded charges (e.g. in an ionized universe):

$$
\phi_{1}=\phi_{2}=4 \ln \left(\frac{E_{e}\left(E_{e}-E_{\gamma}\right)}{E_{\gamma} m_{e}}\right)-2 ;
$$

or 2) neutral hydrogen, in which case the shielding goes to:

$$
\phi_{1} \simeq \phi_{2} \simeq 45
$$

Then, with $E_{e} \gg E_{\gamma}$ we get the result for unshielded charges:

$$
\begin{aligned}
\frac{\mathrm{d} n_{\gamma}}{\mathrm{d} E_{\gamma}} & =\frac{2 n_{\chi} n_{H} \alpha r_{0}^{2} m_{\chi}}{3 C_{e} E_{\gamma}} \frac{4}{3}\left(\frac{6}{m_{e}}+\frac{4}{m_{e}} \ln \left(\frac{m_{e}}{E_{\gamma}}\right)\right. \\
& \left.-\frac{8}{m_{\chi}} \ln \left(\frac{m_{\chi}^{2}}{4 E_{\gamma} m_{e}}\right)\right)
\end{aligned}
$$

and for shielded charges:

$$
\frac{\mathrm{d} n_{\gamma}}{\mathrm{d} E_{\gamma}}=\frac{2 n_{\chi} n_{H} \alpha r_{0}^{2} m_{\chi}}{3 C_{e} m_{e} E_{\gamma}} \frac{4}{3}
$$

We have taken the integration limits from $m_{\chi} / 2$ to $m_{e}$ (strictly speaking the lower limit should be $m_{e}+E_{\gamma}$, but the latter correction is negligible at small $E_{\gamma}$ ). Note that in both cases the spectrum goes as $E_{\gamma}^{-1}$, which does not match the desired shape to reproduce the radio excess. We finally estimate the contribution to other limits. Rewriting the latter in the language of Section 5, and evaluating the spectrum today:

$$
\frac{\mathrm{d} n_{\gamma}}{\mathrm{d} E_{\gamma}}=\frac{3 \alpha n_{\chi, 0} m_{e}}{4 \pi m_{p} z_{d} E_{\gamma}} \frac{\Omega_{B, 0}}{\Omega_{\gamma, 0}}
$$

Comparing this with the CS, this is a very small contribution, even in the $\mathrm{keV}$ region:

$$
\frac{I_{\mathrm{Brems}}}{I_{\mathrm{C}}} \simeq 4 \times 10^{-10}\left(\frac{E_{\gamma}}{\mathrm{eV}}\right)^{1 / 2} .
$$

If injection of electrons instead occurs after reionization, the bremsstrahlung contribution is at most larger by a factor of $2\left(3+2 \ln \left(m_{e} / z_{d} E_{\gamma}\right)\right) \sim 80$ for $z_{d} \sim 10$ and $\nu \sim$ $\mathrm{GHz}$, but is further suppressed at high energies due to the logarithmic terms.
[1] D. J. Fixsen, A. Kogut, S. Levin, M. Limon, P. Lubin, P. Mirel, M. Seiffert and J. Singal et al., Astrophys. J. 734:5, (2011) arXiv:0901.0555 [astro-ph.CO].

[2] R. S. Roger, C. H. Costain, T. L. Landecker and C. M. Swerdlyk, astro-ph/9902213.

[3] A. E. Guzman, J. May, H. Alvarez and K. Maeda, Astron. Astrophys. 525, A138 (2011) [arXiv:1011.4298 [astroph.GA]].

[4] C. G. T. Haslam, et al., Astron. Astrophys. 100, 209 (1981).

[5] P. Reich and W. Reich, Astron. Astrophys. Suppl. 63, 205 (1986).

[6] M. Seiffert, D. J. Fixsen, A. Kogut, S. M. Levin, M. Limon, P. M. Lubin, P. Mirel and J. Singal et al., arXiv:0901.0559 [astro-ph.CO].

[7] A. Kogut, D. J. Fixsen, S. M. Levin, M. Limon, P. M. Lubin, P. Mirel, M. Seiffert and J. Singal et al., Astrophys. J. 734, 4 (2011) [arXiv:0901.0562 [astro-ph.GA]].
[8] J. Singal, L. Stawarz, A. Lawrence and V. Petrosian, Mon. Not. Roy. Astron. Soc. 409, 1172 (2010) [arXiv:0909.1997 [astro-ph.CO]].

[9] T. Vernstrom, D. Scott and J. Wall, arXiv:1102.0814 [astro-ph.CO].

[10] N. Fornengo, R. Lineros, M. Regis and M. Taoso, Phys. Rev. Lett. 107, 271302 (2011) [arXiv:1108.0569 [hep$\mathrm{ph}]$.

[11] N. Fornengo, R. Lineros, M. Regis and M. Taoso, JCAP 1203, 033 (2012) [arXiv:1112.4517 [astro-ph.CO]].

[12] N. Fornengo, R. A. Lineros, M. Regis and M. Taoso, JCAP 1201, 005 (2012) [arXiv:1110.4337 [astro-ph.GA]].

[13] D. Hooper, A. V. Belikov, T. E. Jeltema, T. Linden, S. Profumo and T. R. Slatyer, arXiv:1203.3547 [astroph.CO].

[14] Y. Yang, G. Yang, X. Huang, X. Chen, T. Lu and H. Zong, arXiv:1206.3750 [astro-ph.HE].

[15] G. Holder, arXiv:1207.0856 [astro-ph.CO]. 
[16] S. 'i. Ando and E. Komatsu, Phys. Rev. D 73, 023521 (2006) [astro-ph/0512217].

[17] J. J. Condon, W. D. Cotton, E. B. Fomalont, K. I. Kellermann, N. Miller, R. A. Perley, D. Scott and T. Vernstrom et al., Astrophys. J. 758, 23 (2012) [arXiv:1207.2439 [astro-ph.CO]].

[18] M. G. Hauser and E. Dwek, Ann. Rev. Astron. Astrophys. 39, 249 (2001) [astro-ph/0105539].

[19] X. -L. Chen and M. Kamionkowski, Phys. Rev. D 70, 043502 (2004) [astro-ph/0310473].

[20] T. R. Slatyer, N. Padmanabhan and D. P. Finkbeiner, Phys. Rev. D 80, 043526 (2009) [arXiv:0906.1197 [astroph.CO]].

[21] R. C. Hickox and M. Markevitch, Astrophys. J. 661, L117 (2007) [astro-ph/0702556].

[22] M. Galeazzi, A. Gupta, K. Covey and E. Ursino Astrophys. J. 658, 1081 (2007) [astro-ph/0609528].

[23] G. Bertone, W. Buchmuller, L. Covi and A. Ibarra, JCAP 0711, 003 (2007) [arXiv:0709.2299 [astro-ph]].

[24] J. M. Overduin and P. S. Wesson, Phys. Rept. 402, 267 (2004) [astro-ph/0407207].

[25] R. Durrer, P. G. Ferreira and T. Kahniashvili, Phys. Rev. D 61, 043001 (2000) [astro-ph/9911040].

[26] K. Subramanian and J. D. Barrow, Phys. Rev. D 58, 083502 (1998) [astro-ph/9712083].

[27] A. Mack, T. Kahniashvili and A. Kosowsky, Phys. Rev. D 65, 123004 (2002) [astro-ph/0105504].

[28] A. Lewis, Phys. Rev. D 70, 043011 (2004) [astroph/0406096].

[29] D. G. Yamazaki, K. Ichiki, T. Kajino and G. J. Mathews, Phys. Rev. D 81, 023008 (2010) [arXiv:1001.2012 [astro-
ph.CO]].

[30] D. Paoletti and F. Finelli, Phys. Rev. D 83, 123533 (2011) [arXiv:1005.0148 [astro-ph.CO]].

[31] J. R. Shaw and A. Lewis, Phys. Rev. D 86, 043510 (2012) [arXiv:1006.4242 [astro-ph.CO]].

[32] C. Caprini and R. Durrer, Phys. Rev. D 65, 023517 (2001) [astro-ph/0106244].

[33] F. C. Jones, Phys. Rev. 167, 1159 (1968).

[34] G. R. Blumenthal and R. J. Gould, Rev. Mod. Phys. 42, 237 (1970)

[35] L. Zhang, X. Chen, M. Kamionkowski, Z. -g. Si and Z. Zheng, Phys. Rev. D 76, 061301 (2007) [arXiv:0704.2444 [astro-ph]].

[36] S. Galli, F. Iocco, G. Bertone and A. Melchiorri, Phys. Rev. D 80, 023505 (2009) [arXiv:0905.0003 [astroph.CO]].

[37] J. M. Cline, Z. Liu and W. Xue, Phys. Rev. D 85, 101302 (2012) [arXiv:1201.4858 [hep-ph]].

[38] J. M. Cline, Z. Liu and W. Xue, arXiv:1207.3039 [hep$\mathrm{ph}]$.

[39] S. Davidson, S. Hannestad and G. Raffelt, JHEP 0005 , 003 (2000) [hep-ph/0001179].

[40] J. Jaeckel and A. Ringwald, Ann. Rev. Nucl. Part. Sci. 60, 405 (2010) [arXiv:1002.0329 [hep-ph]].

[41] A. A. Prinz, R. Baggs, J. Ballam, S. Ecklund, C. Fertig, J. A. Jaros, K. Kase and A. Kulikov et al., Phys. Rev. Lett. 81, 1175 (1998) [hep-ex/9804008].

[42] K. Lawson and A. R. Zhitnitsky, arXiv:1210.2400 [astroph.CO]. 